\title{
Absolute spectroscopic factors from nuclear knockout reactions
}

\author{
B. A. Brown, ${ }^{1,2}$ P. G. Hansen, ${ }^{1,2}$ B. M. Sherrill, ${ }^{1,2}$ and J. A. Tostevin ${ }^{3}$ \\ ${ }^{1}$ National Superconducting Cyclotron Laboratory, Michigan State University, East Lansing, Michigan 48824 \\ ${ }^{2}$ Department of Physics and Astronomy, Michigan State University, East Lansing, Michigan 48824 \\ ${ }^{3}$ Department of Physics, School of Physics and Chemistry, University of Surrey, Guildford, Surrey GU2 7XH, United Kingdom
}

(Received 12 December 2001; published 23 May 2002)

\begin{abstract}
Experimental cross sections at high energies for the ${ }^{12} \mathrm{C}\left({ }^{16} \mathrm{O},{ }^{15} \mathrm{~N} / \mathrm{O}\right) X$ and ${ }^{12} \mathrm{C}\left({ }^{12} \mathrm{C},{ }^{11} \mathrm{~B} / \mathrm{C}\right) X$ nucleon removal reactions are reduced by factors $R_{s}=0.49-0.68$ relative to calculations in the shell model and eikonal reaction theory. For protons, this is exactly what has been found in the $\left(e, e^{\prime} p\right)$ reaction. We suggest that nuclear knockout has potential for extending the measurement of precise orbital occupancies to neutron states and to a wide range of nuclei including rare radioactive species. The halo proton in radioactive ${ }^{8} \mathrm{~B}$ turns out to have a stronger presence in the wave function with $R_{s}=0.88(4)$.
\end{abstract}

DOI: 10.1103/PhysRevC.65.061601

PACS number(s): 21.60.Cs, 24.50.+g, 25.60. $-\mathrm{t}, 27.20 .+\mathrm{n}$

The independent-particle shell model has been eminently successful in accounting for the structure of nuclei at low energies. Starting from a picture of particles moving in a potential generated by effective interparticle interactions, Hartree-Fock calculations can to a large extent account for the nuclear binding and shell structure, especially near the closed shells. Away from these it becomes necessary explicitly to take into account the mixing of many valence configurations. This typically involves the diagonalization of a large matrix representing the (effective) interactions in a restricted space of orbitals near the Fermi surface [1]. The shell model has in common with other calculations in many-body quantum theory that the physical particles may differ from those used in the theoretical description. They can have an effective mass [2] and different occupancies, see Pandharipande et al. [3]. To determine the occupancies of single-particle states, one has to measure absolute spectroscopic factors, which presents a longstanding problem. It is only recently that $\left(e, e^{\prime} p\right)$ experiments have shown that the occupancies in a wide range of magic and near-magic nuclei are significantly below those expected from the simple shell model. The systematics [4] suggests a general quenching factor, independent of mass and of the order of 50\%-60\%. It appears [4] that $\left(d,{ }^{3} \mathrm{He}\right)$ transfer cross sections can be reanalyzed to give agreement with this.

In the present work we show that single-nucleon knockout reactions at intermediate and high energies and in inverse kinematics allow a precise assessment of single-particle occupancies, and we extend the analysis to neutron states and to the case of a radioactive nucleus, ${ }^{8} \mathrm{~B}$. The technique, originally developed [5] at energies of 50-70 MeV/nucleon, observes projectile residues in a high-resolution spectrograph. A coincidence with $\gamma$ rays identifies individual final levels. The resulting partial cross sections, analyzed in eikonal reaction theory [6], yield spectroscopic factors, while the shape of the longitudinal momentum distribution determines the orbital angular momentum $l$. A first survey of a number of cases in the $p, s d$ shells $[7,8]$ including data for $\approx 25$ measured cross sections, mostly for weakly bound nucleons, found rather good agreement, but in almost all cases neither experiment nor theory, were accurate enough to reveal the presence of significant rescaling.
A more exacting comparison is offered by several very accurate inclusive cross sections measured at high energy. For the nuclei ${ }^{16} \mathrm{O}$ and ${ }^{12} \mathrm{C}$ an additional attractive feature is that both have been investigated in the $\left(e, e^{\prime} p\right)$ reaction, which today is considered the benchmark for spectroscopic factors. For our third case, radioactive ${ }^{8} \mathrm{~B}$ with a half-life of only $0.8 \mathrm{~s}$, such data are clearly not available. However, ${ }^{8} \mathrm{~B}$ has been the subject of many precise studies and occupies a unique place in nuclear astrophysics. Our three test cases fulfill a number of important criteria.

(i) The projectiles and their residues are all nuclei in the $p$ shell, where the many-body shell model with effective interactions has a high predictive power.

(ii) The existing experimental data are good to $\approx 5 \%$.

(iii) Data were taken at high energies, where the eikonal approximation is expected to be most reliable.

(iv) A wide range in incident energies, here 0.14-2.1 $\mathrm{GeV} /$ nucleon, tests that the spectroscopic factor is extracted consistently.

(v) The selected cases are all for carbon targets for which the Coulomb contributions, which are less well understood, are small.

The analysis in its main lines follows previous work $[5,6,9,10]$ in assuming that the theoretical partial cross section to a given final state $n I^{\pi}$ of the residual nucleus (the core) can be written as

$$
\sigma_{t h}\left(n I^{\pi}\right)=\sum_{j} S_{\text {c.m. }}\left(n I^{\pi}, l j\right) \sigma_{s p}\left(B_{N}, l j\right) .
$$

Here $S_{\text {c.m. }}\left(n I^{\pi}, l j\right)=A /(A-1) S\left(n I^{\pi}, l j\right)$ is the spectroscopic factor with a center-of-mass correction $[11,12]$ included. The quantity $S$ expresses the parentage of the initial state with respect to a specific final state coupled to a nucleon with given angular-momentum quantum numbers $(l j)$, and it has been taken from many-body shell-model calculations [1315].

The $\sigma_{s p}$ are the single-particle removal cross sections, which are strongly dependent on the orbital angular momentum $l$ and on the nucleon separation energy $B_{N}$. They are obtained from expressions given by Hencken et al. [16] and by Tostevin $[6,9]$ as the sum of two incoherent contributions, 
representing mechanisms referred to as stripping (absorption) and diffraction dissociation (elastic breakup). The interactions enter as the elastic $S$ matrices (or profile functions) for the core-target and nucleon-target systems, expressed as functions of the individual impact parameters. In the optical limit of Glauber theory [17], the essential input parameters for the calculation of the profile functions are the free $n n$ and $n p$ cross sections. The interaction range is here represented by a $\delta$ function for energies above $0.3 \mathrm{GeV} /$ nucleon and by Gaussian range parameters $\beta_{n n}=\beta_{n p}=0.5 \mathrm{fm}$ below this energy. Use of this range correction also above $0.3 \mathrm{GeV} /$ nucleon would increase the single-particle cross section for the ${ }^{8} \mathrm{~B}$ case by $1.8 \%$. The real-to-imaginary ratios are set to zero above $0.8 \mathrm{GeV} /$ nucleon and interpolated in the table given by Ray [18] below this energy. The core and target mass distributions are assumed to have Gaussian shapes with rms radii taken from measured interaction cross sections $[17,19]$ and from measured charge radii with the proton charge radius subtracted in quadrature. The value used for the ${ }^{12} \mathrm{C}$ radius was $2.32 \mathrm{fm}$. It will be seen that the singleparticle cross sections emerge from a rigorously defined parameter set. As a test we calculated the reaction cross sections [19] of ${ }^{7} \mathrm{Li},{ }^{12} \mathrm{C}$, and ${ }^{16} \mathrm{O}$ at energies $0.79,0.95$, and $0.97 \mathrm{GeV} /$ nucleon, respectively. The results agree within $4 \%$ or better.

For the composite (nucleon-core) projectiles the wave functions corresponding to the relative motion are calculated in a Woods-Saxon potential, the depth of which is adjusted to reproduce the separation energy of the nucleon for given initial and final states. All the previous calculations of spectroscopic factors [7] used a "standard set" with radius and diffuseness parameters $r_{0}=1.25$ and $a=0.7$. For each of the three projectiles discussed in the present work, analyses exist that permit the selection of an optimized set. However, use of the "standard set" would not change the conclusions appreciably.

We define a quenching factor as the ratio of the experimental inclusive cross sections to the value obtained theoretically

$$
R_{s}=\frac{\sigma_{\text {exp }}}{\sum_{n I^{\pi}} \sigma_{t h}\left(n I^{\pi}\right)}
$$

where the sum is taken over all states that lie below the proton and neutron thresholds in the daughter nuclei. The deviation of $R_{s}$ from unity measures the effect of the shortrange correlations. The input parameters for calculating $\sigma_{t h}\left(n I^{\pi}\right)$ for the three projectiles were selected as follows.

(i) ${ }^{16} \mathrm{O}$. The results of the calculation are given in Table I. The proton (neutron) separation energies to the ground state are $12.13(15.66) \mathrm{MeV}$. The knockout reaction populates predominantly the two states at $0\left(\frac{1}{2}^{-}\right)$and $6.32\left(\frac{3}{2}^{-}\right) \mathrm{MeV}(0$, 6.176 MeV for the mirror nucleus) with theoretical spectroscopic factors $S$ of 1.65 and 3.29, respectively, calculated with the WBP interaction in a $4 \hbar \omega$ model space [20]. Many other states are known up to $10 \mathrm{MeV}$, but the $(p, d)$ reaction [21] gave only small spectroscopic factors to these, less than
TABLE I. Cross sections for the reactions ${ }^{12} \mathrm{C}\left({ }^{12} \mathrm{C},{ }^{11} \mathrm{~B}\right) X,{ }^{12} \mathrm{C}\left({ }^{12} \mathrm{C},{ }^{11} \mathrm{C}\right) X,{ }^{12} \mathrm{C}\left({ }^{16} \mathrm{O},{ }^{15} \mathrm{~N}\right) X$ and ${ }^{12} \mathrm{C}\left({ }^{16} \mathrm{O},{ }^{15} \mathrm{O}\right) X$.

\begin{tabular}{|c|c|c|c|c|c|c|}
\hline \multirow[t]{2}{*}{${ }^{A-1} Z$} & \multirow{2}{*}{$\begin{array}{c}E_{B} \mathrm{MeV} / \\
\text { nucleon }\end{array}$} & \multirow[t]{2}{*}{$E^{*}$} & $\sigma_{s p}(\mathrm{mb})^{\mathrm{a}}$ & \multirow{2}{*}{$\begin{array}{c}\sigma_{t h} \\
(\mathrm{mb})\end{array}$} & \multicolumn{2}{|l|}{$\sigma_{\text {expt }}$} \\
\hline & & & Strip. Diffr. & & $(\mathrm{mb})$ & $R_{s}$ \\
\hline
\end{tabular}

\begin{tabular}{cccccccc}
${ }^{11} \mathrm{~B}$ & 250 & $\mathrm{a}$ & 21.9 & 1.8 & 100.5 & $65.6(26)$ & $0.65(3)$ \\
& 1050 & $\mathrm{a}$ & 20.8 & 1.9 & 96.1 & $48.6(24)^{\mathrm{c}}$ & $0.51(3)$ \\
& 2100 & $\mathrm{a}$ & 20.6 & 2.0 & 96.1 & $53.8(27)^{\mathrm{c}}$ & $0.56(3)$ \\
\hline
\end{tabular}

$\begin{array}{llllllll}{ }^{11} \mathrm{C} & 250 & \mathrm{a} & 21.4 & 1.7 & 98.2 & 56.0(41)^{\mathrm{b}} & 0.57(4)\end{array}$

$\begin{array}{lllllll}1050 & \text { a } & 20.2 & 1.8 & 93.4 & 44.7(28)^{\mathrm{c}} & 0.48(3)\end{array}$

$\begin{array}{lllllll}2100 & \text { a } & 20.1 & 1.9 & 93.3 & 46.5(23)^{\mathrm{c}} & 0.50(3)\end{array}$

$\begin{array}{cccccccc}{ }^{15} \mathrm{~N} & 2100 & 0 & 15.40 & 1.77 & & & \\ & & 6.324 & 12.95 & 1.30 & & & \\ & \text { Sum } & & & 80.2 & 54.2(29)^{\mathrm{b}} & 0.68(4) \\ & & & \end{array}$

$\begin{array}{cccccccc}{ }^{15} \mathrm{O} & 2100 & 0 & 14.63 & 1.61 & & & \\ & & 6.176 & 12.54 & 1.23 & & & \\ & & \text { Sum } & & & 76.9 & 42.9(23)^{\mathrm{c}} & 0.56(3)\end{array}$

a The single-particle cross sections are those for the ground state. The values for the two excited states are 5-6\% smaller. The $\sigma_{t h}$ listed are the sum of all contributions. The energies and spectroscopic factors are given in the text.

${ }^{\mathrm{b}}$ Reference [25].

${ }^{\mathrm{c}}$ Reference [23].

$10 \%$ of the total. The preferred radius and diffuseness parameters are [22] $r_{0}=1.311$ and $a=0.534$, but the "standard set" would have given essentially the same results. The matter radii of ${ }^{15} \mathrm{~N}$ and ${ }^{15} \mathrm{O}$ were taken to be $2.44 \mathrm{fm}$ [19]. The cross sections for the inclusive ${ }^{12} \mathrm{C}\left({ }^{16} \mathrm{O},{ }^{15} \mathrm{~N} / \mathrm{O}\right) X$ knockout reaction have been measured at the $\mathrm{LBL}$ at $2.1 \mathrm{GeV} /$ nucleon [23]. The difference between $R_{s}=0.56(3)$ for neutron removal against 0.68(4) for proton removal could arise from an excess of cross section between 7 and $11 \mathrm{MeV}$ corresponding to the nucleon thresholds for the two residues. If this is the case, the smaller value, which corresponds to the lower threshold, would actually be the best estimate.

(ii) ${ }^{12} \mathrm{C}$. The results of the calculation are given in Table I. The proton (neutron) separation energies are 15.96 (18.72) $\mathrm{MeV}$ with the knockout leading predominantly to states at 0

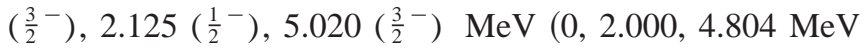
for the mirror nucleus) all of which are below the nucleon decay thresholds. The theoretical spectroscopic factors $S$ calculated with the WBP interaction [15] are 3.16, 0.58, and 0.19 . The small remainder of 0.07 is fragmented over many states above $10 \mathrm{MeV}$ in excitation. These values agree well with other $p$-shell calculations. The second and fourth moments of the ${ }^{12} \mathrm{C}$ charge radii have been used by Bassel et al. [24] to fix the radius and diffuseness parameters of the Woods-Saxon potential to $r_{0}=1.310$ and $a=0.55$. Use of the "standard" set would have given the same knockout cross sections to within $0.5 \%$. The matter radii of ${ }^{11} \mathrm{~B}$ and ${ }^{11} \mathrm{C}$ 
were taken to be $2.11 \mathrm{fm} \mathrm{[19].} \mathrm{The} \mathrm{cross} \mathrm{sections} \mathrm{for} \mathrm{the}$ inclusive ${ }^{12} \mathrm{C}\left({ }^{12} \mathrm{C},{ }^{11} \mathrm{~B} / \mathrm{C}\right) X$ knockout reaction have been measured at LBL at the energies 0.25 [25], 1.05 and 2.1 $\mathrm{GeV} /$ nucleon [23]. All measurements are precise to 5\%-8\%. The results show a strong quenching of the single-particle strength and the approximate symmetry between the two product nuclei that follows from isospin conservation. The rather high values at $0.25 \mathrm{GeV} /$ nucleon may reflect an experimental problem, and we have chosen to represent the data by the average of the measurements at 1.05 and 2.1 $\mathrm{GeV}$. For ${ }^{11} \mathrm{~B}$ we then have $R_{s}=0.53(2)$ and for ${ }^{11} \mathrm{C}, R_{s}$ $=0.49(2)$.

These results are based on a sampling of the singleparticle wave function near and beyond the nuclear surface. The (average) outer fraction of the single-particle state that is accessible in the reaction may be estimated as the ratio of $9 \%$ of the calculated cross section for proton stripping to the total reaction cross section of free protons. (The localization of the nucleon knockout to the surface region also shows up in a very characteristic way in the parallel-momentum distributions of the heavy-ion residues [26].)

The experimental spectroscopic factors for nuclear proton knockout on ${ }^{16} \mathrm{O}$ and ${ }^{12} \mathrm{C}$ are identical to those obtained in the $\left(e, e^{\prime} p\right)$ reaction, see the summary by Kramer et al. [4] and earlier works [27-29]. Recalculated on the basis of our theoretical spectroscopic factors, which are very similar to theirs, the results for $R_{s}$ for oxygen and carbon are $0.67(5)$ and $0.51(3)$, respectively, in excellent agreement with our values of $0.68(4)$ and $0.53(2)$. The corresponding factors in the neutron-removal channel, for which there are no previous measurements, are 0.56(3) and 0.49(2). We conclude that the two techniques measure the same quantity and that we have (i) confirmed the quenching of the proton occupancy in these nuclei by an approach that is essentially parameter free, and (ii) extended the results to neutron occupancies.

(iii) ${ }^{8} \mathrm{~B}$. The results of the calculation are given in Table II. The proton separation energy is $0.1375(10) \mathrm{MeV}$. The theoretical spectroscopic factors $S$ given by Brown et al. [12] for the PJT interaction [1] are to the $\frac{3}{2}^{-}$ground state of ${ }^{7} \mathrm{Be} 0.97\left(p_{3 / 2}\right)$ and $0.06\left(p_{1 / 2}\right)$. To the $\frac{1}{2}^{-}$excited state at $0.429 \mathrm{MeV}$ state the value is $0.22\left(p_{3 / 2}\right)$. These values agree to within 5\% with those from other interactions appropriate for the lower part of the $p$ shell [12]. The Coulomb displacement energy in the $A=8$ isospin triplet served [12] to fix the radius and diffuseness parameters of the Woods-Saxon potential to $r_{0}=1.254$ and $a=0.62$. If the "standard set" had been used, the knockout cross sections would have increased by $7.5 \%$, and $R_{s}$ would have decreased by the same amount. The matter radius of ${ }^{7} \mathrm{Be}$ was taken to be $2.31 \mathrm{fm}$ [19]; with the value $2.24 \mathrm{fm}$ deduced from the ${ }^{7} \mathrm{Li}$ charge radius, the single-particle cross sections would increase by $2 \%$. The contributions from Coulomb dissociation given in Table II have been calculated for us by Typel [30]. They are seen to be a small correction. Experimental cross sections for the inclusive ${ }^{12} \mathrm{C}\left({ }^{8} \mathrm{~B},{ }^{7} \mathrm{Be}\right) \mathrm{X}$ knockout reaction have been obtained at the GSI [31-33] at energies ranging from 0.14 to $1.44 \mathrm{GeV} /$ nucleon and with a precision that is better than 5\% in most cases. The results for the quenching factor are consistent over the full energy range and correspond to $R_{S}$
TABLE II. Cross sections for the reaction ${ }^{12} \mathrm{C}\left({ }^{8} \mathrm{~B},{ }^{7} \mathrm{Be}\right) X$.

\begin{tabular}{|c|c|c|c|c|c|c|c|}
\hline \multirow{2}{*}{$\begin{array}{l}E_{B} \\
\mathrm{MeV} / \text { nucleon }\end{array}$} & \multirow{2}{*}{$\begin{array}{c}E^{*} \\
\mathrm{MeV}\end{array}$} & \multicolumn{3}{|c|}{$\sigma_{s p}(\mathrm{mb})$} & \multirow{2}{*}{$\begin{array}{l}\sigma_{t h}^{\mathrm{a}} \\
(\mathrm{mb})\end{array}$} & \multicolumn{2}{|c|}{$\sigma_{\text {expt }}$} \\
\hline & & Str. & Dif. & Cou. & & $(\mathrm{mb})$ & $R_{s}$ \\
\hline \multirow[t]{3}{*}{142} & 0 & 59.8 & 26.6 & 4.0 & 107.1 & & \\
\hline & 0.429 & 53.6 & 20.6 & 1.5 & 19.0 & & \\
\hline & Sum: & & & & 126.1 & $109(1)^{b}$ & $0.86(1)$ \\
\hline \multirow[t]{3}{*}{285} & 0 & 57.3 & 11.9 & 2.6 & 85.0 & & \\
\hline & 0.429 & 51.8 & 9.2 & 1.0 & 15.6 & & \\
\hline & Sum: & & & & 100.6 & $89(2)^{b}$ & $0.88(2)$ \\
\hline \multirow[t]{3}{*}{936} & 0 & 59.4 & 14.5 & 1.6 & 89.4 & & \\
\hline & 0.429 & 52.8 & 11.1 & 0.6 & 16.2 & & \\
\hline & Sum & & & & 105.6 & $94(9)^{\mathrm{c}}$ & $0.89(9)$ \\
\hline \multirow[t]{3}{*}{1440} & 0 & 60.5 & 15.9 & 1.4 & 92.1 & & \\
\hline & 0.429 & 53.6 & 12.1 & 0.6 & 16.7 & & \\
\hline & Sum: & & & & 108.8 & $96(3)^{d}$ & $0.88(3)$ \\
\hline
\end{tabular}

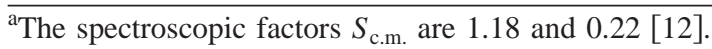

${ }^{\mathrm{b}}$ Reference [31], statistical errors only.

${ }^{\mathrm{c}}$ Reference [32].

${ }^{\mathrm{d}}$ Reference [33], weighted average of two reported values of 94(4) and $100(5)$.

$=0.88$ (4) (an unweighted average, error suggested by us). The smaller proton separation energy $(0.14 \mathrm{MeV})$ may be behind this difference from the ${ }^{16} \mathrm{O}$ and ${ }^{12} \mathrm{C}$ results. It would clearly be interesting, although technically very difficult, to obtain $\left(e, e^{\prime} p\right)$ data for loosely bound radioactive nuclei. The ${ }^{8} \mathrm{~B}$ reaction is less surface dominated than that of ${ }^{16} \mathrm{O}$ and ${ }^{12} \mathrm{C}$; it samples an average of $25 \%$ of the outer proton probability.

Our analysis assumes that the theory correctly predicts the relative cross sections to the two levels of ${ }^{7} \mathrm{Be}$. A recent experiment by Cortina-Gil et al. [32] demonstrates that this is correct. They measured the $\gamma$ rays from the $0.429 \mathrm{MeV}$ level and found that this branch is $13(3) \%$. The theoretical calculation of Table II gives $15 \%$.

The ${ }^{8} \mathrm{~B}$ results provide a connection to the $S_{17}$ value for the ${ }^{7} \mathrm{Be}(p, \gamma)$ reaction, well known for its importance in solar physics. In an interesting paper, Trache et al. [34] start from the assumption that knockout reactions furnish absolute occupancies, a viewpoint that finds support in the present work. They analyze experimental data for the $\left({ }^{8} \mathrm{~B},{ }^{7} \mathrm{Be}\right)$ cross section for a number of energies and targets and extract the square of the asymptotic normalization coefficient $C$ for the odd-proton $p$ state wave function expressed in terms of the asymptotically correct Whittaker function. Since the knockout cross section is weighted towards the nuclear surface, it is correlated with the asymptotic normalization. Thus the deduced asymptotic normalization depends less on the potential than does the spectroscopic factor. With the parameters of the present work and evaluating the partial derivatives with respect to the Woods-Saxon parameters we find $\delta\left(C^{2}\right) / C^{2}$ 
$=0.24 \delta r_{0}+0.59 \delta a$, so that typical errors arising from the shape parameters are only $2 \%-3 \%$. (The corresponding expression for the spectroscopic factor has the coefficients -0.52 and $-0.95 \mathrm{fm}^{-1}$.) Our result is $C^{2}=0.57(4)$, well above the value 0.45(4) obtained in [34]. Part of the apparent discrepancy may arise from their use of data that have substantial Coulomb contributions; already for a Si target the Coulomb cross section contributes $\approx 25 \%$. There are also differences in the calculated nuclear cross sections. For the first two entries in Table II, we obtain $C^{2}$ values that differ by $2 \%$, where Trache et al. find a $24 \%$ difference. If the experimental $R_{s}$ is included in the analysis by Brown et al. [12], their value is modified to $S_{17}=21.2(13) \mathrm{eV}$ b. This assumes that a single-particle potential model is adequate for extracting $S_{17}$, which is not necessarily the case. Direct measurements of the electromagnetic transition rate avoid this issue. From electrodissociation a somewhat lower value of $17.8(+1.4 /-1.2)$ eV b was found by Davids et al. [35] and also in other works cited therein, while a recent remeasurement of the $(p, \gamma)$ cross section [36] gave a value of $22.3(0.7)(0.5)$.

In summary, the analysis presented here strongly suggests that nucleon knockout reactions at intermediate and high energies have the power to provide absolute spectroscopic factors for both neutrons and protons and will allow us to explore the foundations of the shell model in a systematic way. Furthermore the technique is relatively simple, and it is very sensitive having already been applied [10] to incident beams of less than one atom per second. It will give access to all nuclei that can be produced as radioactive beams, not just to those available as stable targets. The results for proton knockout from ${ }^{12} \mathrm{C}$ and ${ }^{16} \mathrm{O}$ give quenching ratios $R_{s}$ that are about one-half, in agreement with what is found in the $\left(e, e^{\prime} p\right)$ reaction. Our results for neutron removal confirm this for the first time. For the case of the $l=1$ proton halo of ${ }^{8} \mathrm{~B}$, for which no electron data are available, we find a result that is much closer to the full shell-model strength. This suggests that the picture of a universal quenching factor close to 0.5 for all nuclei independent of mass [4] may not be the full story. (Independently of this, one would intuitively expect a well-developed halo state to attain the limit of $R_{S}$ $=1$.) More data, especially on unstable nuclei are needed. Another open question is the quantitative accuracy of our approach in the experimentally attractive energy region of 50-100 MeV/nucleon. This also calls for more precise experiments.

Stimulating discussions with Sam M. Austin and Thomas Baumann are gratefully acknowledged. This work was supported by the National Science Foundation under Grant Nos. PHY 9528844 and PHY 0070911 and by the United Kingdom Engineering and Physical Sciences Research Council (EPSRC) Grant No. GR/M82141.
[1] B.A. Brown, Prog. Part. Nucl. Phys. 47, 517 (2001).

[2] G.F. Bertsch and T.T.S. Kuo, Nucl. Phys. A112, 204 (1968).

[3] V.R. Pandharipande, I. Sick, and P.K.A. deWitt Huberts, Rev. Mod. Phys. 69, 981 (1997).

[4] G.J. Kramer, H.P. Blok, and L. Lapikas, Nucl. Phys. A679, 267 (2001).

[5] A. Navin et al., Phys. Rev. Lett. 81, 5089 (1998).

[6] J.A. Tostevin, J. Phys. G 25, 735 (1999).

[7] P.G. Hansen and B.M. Sherrill, Nucl. Phys. A693, 133 (2001).

[8] J. Enders et al., Phys. Rev. C 65, 034318 (2002).

[9] J.A. Tostevin, Nucl. Phys. A682, 320c (2001).

[10] V. Maddalena et al., Phys. Rev. C 63, 024613 (2001).

[11] A.E.L. Dieperink and T. de Forest, Phys. Rev. C 10, 543 (1974)

[12] B.A. Brown, A. Csoto, and R. Sherr, Nucl. Phys. A597, 66 (1996).

[13] B.A. Brown, in International School of Heavy-Ion Physics, edited by R.A. Broglia and P.G. Hansen, 4th Course: Exotic Nuclei (World Scientific, Singapore, 1998), p. 1.

[14] B.A. Brown and B.H. Wildenthal, Annu. Rev. Nucl. Part. Sci. 38, 29 (1988).

[15] E.K. Warburton and B.A. Brown, Phys. Rev. C 46, 923 (1992).

[16] K. Hencken, G.F. Bertsch, and H. Esbensen, Phys. Rev. C 54, 3043 (1996).
[17] J.S. Al-Khalili, J.A. Tostevin, and I.J. Thompson, Phys. Rev. C 54, 1843 (1996).

[18] L. Ray, Phys. Rev. C 20, 1857 (1979).

[19] A. Ozawa, T. Suzuki, and I. Tanihata, Nucl. Phys. A693, 32 (2001).

[20] E.K. Warburton, B.A. Brown, and D.J. Millener, Phys. Lett. B 293, 7 (1992).

[21] J.L. Snelgrove and E. Kashy, Phys. Rev. 187, 1246 (1969).

[22] B.A. Brown et al., Phys. Rev. C 26, 2247 (1982).

[23] D.L. Olson et al., Phys. Rev. C 28, 1602 (1983).

[24] R.H. Bassel et al., J. Phys. G 8, 1215 (1982).

[25] J.M. Kidd et al., Phys. Rev. C 37, 2613 (1988).

[26] P.G. Hansen, Phys. Rev. Lett. 77, 1016 (1996).

[27] G. van der Steenhoven et al., Nucl. Phys. A480, 547 (1988).

[28] M. Leuschner et al., Phys. Rev. C 49, 955 (1994).

[29] A.E.L. Dieperink and P.K.A. de Witt Huberts, Annu. Rev. Nucl. Part. Sci. 40, 239 (1990).

[30] S. Typel (private communication).

[31] B. Blank et al., Nucl. Phys. A624, 242 (1997).

[32] D. Cortina-Gil et al., Phys. Lett. B 529, 36 (2002).

[33] D. Cortina-Gil et al., Eur. Phys. J. A 10, 49 (2001); W. Schwab et al., Z. Phys. A 350, 283 (1995).

[34] L. Trache, F. Carstoiu, C. A. Gagliardi, and R. E. Tribble, Phys. Rev. Lett. 88, 041101 (2002).

[35] B. Davids et al., Phys. Rev. C 63, 065806 (2001).

[36] A.R. Junghans et al., Phys. Rev. Lett. 87, 271102 (2001). 